\title{
Intensive Smoking Cessation Counseling for Patients With Cancer
}

Adam O. Goldstein, MD, MPH; Kimberly A. Shoenbill, MD, PhD; Trevor A. Jolly, MBBS

Smoking remains the leading cause of preventable death in the United States. ${ }^{1,2}$ Tobacco use increases the risk of multiple cancers, including lung, oropharyngeal, pancreas, bladder, stomach, and colon. ${ }^{3}$ Continued tobacco use following a cancer di- $\leftarrow$
Related article page 1406 agnosis increases the risk of cancer recurrence, new primary cancers, and adverse

treatment-related outcomes, including postoperative pulmonary complications, poor surgical healing, and decreased response to chemotherapeutic drugs. ${ }^{4}$

In contrast, tobacco cessation after cancer diagnosis is often associated with improved quality of life, fewer complications related to cancer treatment, and longer survival. ${ }^{4}$ In a 2018 study, the prevalence of cigarette smoking among adult cancer survivors was $11.8 \%,{ }^{5}$ and many cancer survivors who quit smoking after a diagnosis may resume smoking. ${ }^{6}$ Failure to address the unique challenges of cessation contributes to lack of success in initial cancer treatment due to ongoing smoking and has been associated with an estimated incremental cost of nearly $\$ 11000$ per year per smoking patient. ${ }^{7}$

Tobacco abstinence is the strongest predictor of cancer survival, after cancer type and stage at the time of diagnosis. ${ }^{8}$ While guidelines from the National Comprehensive Cancer Network recommend cessation counseling and pharmacologic treatment for patients with cancer who are interested in quitting smoking, ${ }^{4}$ a survey of 1153 physicians of the International Association for the Study of Lung Cancer found that only $44 \%$ reported treating their patients' tobacco dependence. ${ }^{9}$ Barriers to cessation treatment in oncology include time constraints and lack of tobacco cessation training. ${ }^{10}$ A 2019 metaanalysis of randomized clinical trials of tobacco cessation among patients with cancer found no consistently effective interventions, despite similar studies showing efficacy in the general population. ${ }^{11}$

It is in this context that the study by Park et $\mathrm{al}^{12}$ in this issue of JAMA provides an important contribution to the oncology literature. In this unblinded study conducted at $2 \mathrm{Na}$ tional Cancer Institute (NCI) Comprehensive Cancer Centers, the authors randomly assigned 303 patients with newly diagnosed cancer who smoked cigarettes to an intensive treatment group, with 4 weekly, 4 biweekly, and 3-monthly smoking cessation telephone counseling sessions coupled with 12 weeks of free cessation medication (either nicotine replacement therapy, bupropion, or varenicline) or to a standard treatment group, with 4 weekly telephone counseling sessions and medication advice, without provision of free medication. Intention-to-treat analysis of the 221 patients who completed the study (78.1\% completion rate) showed that patients in the intensive treatment group achieved biochemically confirmed, higher 7-day abstinence rates at 6-month follow-up (34.5\%) compared with patients in the standard treatment group (21.5\%). While the $13 \%$ absolute between-group difference was slightly less than hypothesized, this difference may be clinically meaningful.

This theory-driven research provides evidence that intensive smoking counseling over at least 6 months contributes to an effective approach to smoking cessation in patients with cancer. Patients in the intensive counseling group completed a median of 8 counseling sessions compared with a median of 4 sessions in the standard treatment group, and in multivariate models, medications had little influence on effect size estimates. Approximately $30 \%$ of patients $(22 / 74)$ who had quit smoking at 3 months relapsed by 6 months, whereas $35 \%$ of patients (28/80) still smoking at 3 months had quit by 6 months, resulting in modest additional quitting gains (34.5\% vs $31.1 \%$ ) at 6 months. The therapists who provided the counseling had expert training in motivational interviewing, cognitivebased therapy, stress reduction, and cancer-specific support.

The study by Park et $\mathrm{al}^{12}$ has a number of limitations, as acknowledged by the authors. The study was conducted as a randomized clinical trial, in which patients received free counseling and medication, and they were rewarded financially for study procedures (up to $\$ 200$ for baseline, follow-up surveys, and salivary/expired air specimens), which might have served as an unintended incentive for overall participation or abstinence, although this would be expected to be balanced between treatment groups. It took the investigators almost 4 years to recruit the patients, and most of those screened for eligibility declined participation, suggesting potential barriers to broad uptake that warrant future implementation research. Patients and clinicians were not blinded to treatment, patients with lower literacy levels were mostly excluded, and the study was conducted in only 2 cancer centers, limiting generalizability. In addition, the study did not measure cessation rates beyond 6 months, and relapse, even after 6 months of successful quitting, is not infrequent. ${ }^{6}$

The study was not designed to assess the potential effects of e-cigarette use despite its frequent use in the intensive treatment group (17.7\%) and the standard treatment group (19.5\%). e-Cigarette use has increased among cancer survivors in the last decade. In a recent study of 1527 cancer survivors, $3.8 \%$ used e-cigarettes, and $25 \%$ of cancer survivors with complete information on product use $(n=159)$ reported dual use of tobacco products. ${ }^{13}$ Interventions that assess and address e-cigarette and dual use among patients with cancer should be undertaken.

Identification of a dose-response relationship of counseling, alone or in combination with medication, would be 
valuable in identifying the most effective and economical intervention in patients with cancer. While free counseling and medication are not routinely available in the United States for most patients with cancer who smoke, recent funding from the Cancer Center Cessation Initiative has offered short-term support for cessation therapy at NCI Comprehensive Cancer Centers. ${ }^{14}$ Most oncologists are not likely to provide the intensive counseling needed (average of 40 minutes for the first visit in this study and 20 minutes for follow-up visits), but trained smoking cessation therapists can work collaboratively with oncologists to fill this void.

Additional research and data are necessary for more effective smoking cessation programs at cancer centers. To fill this gap, multiple NCI Cancer Centers have recently conducted quality assessment and improvement projects that have shown how system changes in cancer care improve cessation outcomes, including improved documentation of tobacco use; electronic health record optimizations to improve treatment referrals and follow-up; clinician education of treatment options available at their institutions; and inclusion of family members in cessation counseling. ${ }^{15,16}$ Opt-out referral processes, in which patients with cancer who smoke are automatically given appointments with tobacco treatment counselors, are an increasingly important part of a comprehensive program. ${ }^{17}$

Future research on cancer and tobacco cessation should also build on the study by Park et $\mathrm{al}^{12}$ by looking at cessation among patients with cancer who have multiple disparities, including lack of insurance, limited financial resources, those further out from their initial cancer diagnosis, and those who receive care at more rural or community-based oncology facilities. Research among younger cancer survivors who continue to smoke is also particularly important because these patients were less likely to continue treatment in the study by Park et $\mathrm{al}^{12}$ and smoking rates are higher (20.4\%) in younger cancer survivors (18-44 years). ${ }^{5}$

Cancer centers and clinicians who provide care for patients with cancer should go beyond measuring universal tobacco screening and offers of counseling; they must improve on ways to expand the scope of intensive counseling that is integrated into routine cancer care processes. Because the current study showed that greater numbers of counseling sessions as well as booster sessions extending out to 6 months led to a higher likelihood of quitting in the intensive group, therapists in cancer programs should strive to achieve both metrics. Currently, in many centers, only a minority of patients referred for counseling receive such counseling, ${ }^{15,18,19}$ and advanced cessation programs may struggle to provide 4 or more patient counseling sessions. ${ }^{15}$

Payers and health system leaders must acknowledge that current fee-for-service models for cancer treatment are incongruent with models of sustainable intensive smoking cessation counseling. Reimbursement for Medicare, Medicaid, and most private insurers only pays for 4 counseling sessions per quit attempt. ${ }^{20}$ Reimbursement in the fee-for-service model is based on evaluation and management physician billing codes for treatment, rather than certified tobacco treatment specialists who provide intensive counseling and use low reimbursement preventive counseling codes. Such codes do not incentivize additional counseling, and they do not reimburse at levels to sustain intensive programs. Given the cost-effectiveness of smoking cessation for patients with cancer and the consequences of failed treatment, value- and incentive-based reimbursements would potentially engender greater uptake of more intensive counseling from trained cessation therapists. If payers are willing to reimburse cancer centers thousands of dollars per patient annually for cancer treatments, they should be willing to invest in intensive cessation coverage, which ultimately lowers cost and improves outcomes.

Implementing intensive cessation counseling requires more than formulaic algorithms of treatment protocols. System-level change supported by patients, clinician groups, and cancer centers must occur, with provision of resources from payers and health care systems. In such an environment, clinicians can call on the best evidence from well-designed studies and use the art of medicine: the connection and empathy that foster patient trust and tailor treatments to each individual, devoid of stigma and judgment.

\section{ARTICLE INFORMATION}

Author Affiliations: Lineberger Comprehensive Cancer Center, University of North Carolina School of Medicine, Chapel Hill (Goldstein, Jolly); Department of Family Medicine, University of North Carolina School of Medicine, Chapel Hill (Goldstein, Shoenbill); Division of Oncology, Department of Medicine, University of North Carolina School of Medicine, Chapel Hill (Jolly).

Corresponding Author: Adam O. Goldstein, MD, MPH, Department of Family Medicine, University of North Carolina School of Medicine, Manning Drive, CB 7595, Chapel Hill, NC 27599

(aog@med.unc.edu).

Conflict of Interest Disclosures: None reported.

\section{REFERENCES}

1. Reitsma MB, Fullman N, Ng M, et al; GBD 2015 Tobacco Collaborators. Smoking prevalence and attributable disease burden in 195 countries and territories, 1990-2015: a systematic analysis from the Global Burden of Disease Study 2015. Lancet. 2017;389(10082):1885-1906. doi:10.1016/SO140 6736(17)30819-X

2. US Department of Health and Human Services. The Health Consequences of Smoking-50 Years of Progress: A Report of the Surgeon General. US Dept of Health and Human Services, Centers for Disease Control and Prevention: National Center for Chronic Disease Prevention and Health Promotion, Office on Smoking and Health; 2014.

3. US Department of Health and Human Services. Smoking Cessation: A Report of the Surgeon General - Key Findings. US Dept of Health and Human Services, Centers for Disease Control and Prevention, National Center for Chronic Disease Prevention and Health Promotion, Office on Smoking and Health; 2020.

4. Shields PG, Herbst RS, Arenberg D, et al. Smoking cessation, version 1.2016: NCCN clinical practice guidelines in oncology. J Natl Compr Canc
Netw. 2016;14(11):1430-1468. doi:10.6004/jnccn. 2016.0152

5. National Cancer Institute. Cancer Trends Progress Report. Updated March 2020. Accessed September 13, 2020. https://progressreport.cancer. gov/after/smoking

6. Paul CL, Tzelepis F, Boyes AW, D'Este C, Sherwood E, Girgis A. Continued smoking after a cancer diagnosis: a longitudinal study of intentions and attempts to quit. J Cancer Surviv. 2019;13(5): 687-694. doi:10.1007/s11764-019-00787-5

7. Warren GW, Cartmell KB, Garrett-Mayer $E$, Salloum RG, Cummings KM. Attributable failure of first-line cancer treatment and incremental costs associated with smoking by patients with cancer. JAMA Netw Open. 2019;2(4):e191703. doi:10.1001/ jamanetworkopen.2019.1703

8. Karam-Hage M, Cinciripini PM, Gritz ER. Tobacco use and cessation for cancer survivors: an overview for clinicians. CA Cancer J Clin. 2014;64(4):272-290. doi:10.3322/caac.21231 
9. Warren GW, Dibaj S, Hutson A, Cummings KM, Dresler C, Marshall JR. Identifying targeted strategies to improve smoking cessation support for cancer patients. J Thorac Oncol. 2015;10(11): 1532-1537. doi:10.1097/JTO.0000000000000659

10. Rojewski AM, Bailey SR, Bernstein SL, et al. Considering systemic barriers to treating tobacco use in clinical settings in the United States. Nicotine Tob Res. 2019;21(11):1453-1461. doi:10.1093/ntr/nty123

11. Sheeran $P$, Jones $K$, Avishai $A$, et al. What works in smoking cessation interventions for cancer survivors? a meta-analysis. Health Psychol. 2019;38 (10):855-865. doi:10.1037/hea0000757

12. Park ER, Perez GK, Regan S, et al. Effect of sustained smoking cessation counseling and provision of medication vs shorter-term counseling and medication advice on smoking abstinence in patients recently diagnosed with cancer: a randomized clinical trial. JAMA. Published October 13, 2020. doi:10.1001/jama.2020.14851
13. Salloum RG, Huo J, Lee JH, et al. Tobacco and e-cigarette use among cancer survivors in the United States. PLoS One. 2019;14(12):e0226110. doi:10.1371/journal.pone.0226110

14. Croyle RT, Morgan GD, Fiore MC. Addressing a core gap in cancer care: the $\mathrm{NCl}$ Moonshot program to help oncology patients stop smoking. NEngl J Med. 2019;380(6):512-515. doi:10.1056/NEJMp1813913

15. Meyer C, Mitra S, Ruebush E, Sisler L, Wang K, Goldstein AO. A lean quality improvement initiative to enhance tobacco use treatment in a cancer hospital. Int J Environ Res Public Health. 2020;17(6): E2165. doi:10.3390/ijerph17062165

16. Ruebush E, Mitra S, Meyer C, Sisler L, Goldstein AO. Using a family systems approach to treat tobacco use among cancer patients. Int J Environ Res Public Health. 2020;17(6):E2050. doi:10.3390/ ijerph17062050

17. Gali K, Pike B, Kendra MS, et al. Integration of tobacco treatment services into cancer care at
Stanford Int J Environ Res Public Health. 2020:17 (6):E2101. doi:10.3390/ijerph17062101

18. Jose T, Ohde JW, Hays JT, Burke MV, Warner DO. Design and pilot implementation of an electronic health record-based system to automatically refer cancer patients to tobacco use treatment. Int J Environ Res Public Health. 2020;17 (11):E4054. doi:10.3390/ijerph17114054

19. LeLaurin JH, Dallery J, Silver NL, et al. An implementation trial to improve tobacco treatment for cancer patients: patient preferences, treatment acceptability and effectiveness. Int J Environ Res Public Health. 2020;17(7):E2280. doi:10.3390/ ijerph17072280

20. American Lung Association. Tobacco cessation treatment: what is covered? Updated July 21, 2020. Accessed September 12, 2020. https://www.lung. org/policy-advocacy/tobacco/cessation/tobaccocessation-treatment-what-is-covered 